\title{
Leakage Power Consumption of Address Register Interfacing with Different Families of FPGA
}

\author{
Bishwajeet Pandey, Keshav Kumar, Shabeer Ahmad, Amit K Pandit, Deepa Singh, D M Akbar \\ Hussain
}

\begin{abstract}
In this paper, we are designing an address register which is sensitive towards rising in voltage. We analysed the power variation of address register on Xilinx 14.1 ISE Design Suite and the code of address register is written in Verilog hardware description language. In this paper, we have used two FPGA of two different families, one is of Virtex family which is Virtex 6 and the other is of Spartan family which is Spartan 6, to study the power consumption of address register. We have observed the different on chips power which are consumed by address register by varying the voltage from $0.75 \mathrm{~V}$ to $2 \mathrm{~V}$ for Virtex 6 FPGA and $0.75 \mathrm{~V}$ to $3 \mathrm{~V}$ for Spartan 6 FPGA and we observed that when we lower the voltage, lower will be the power consumption. At $2 \mathrm{~V}$, Virtex 6 FPGA stops working and the interface of address register with FPGA burns out. For Spartan 6 FPGA, the same happens at $3 V$ voltage.
\end{abstract}

Keywords-Power variation, Xilinx, Verilog, Voltage Scaling, FPGA.

\section{INTRODUCTION}

Today's world is facing a very enormous problem with the energy crisis. Day by day the demand of people towards natural resources i.e. nonrenewable resources is increasing rapidly. The three main causes of this energy crisis are overconsumption, overpopulation and poor infrastructure [1]. To ensure the less wastage of the natural resources of the planet, we are making an address register which will consume low power in the communication system. As our address register will consume low power, there will be less use of electricity and thus less use of natural resources of the planet. The design of address register is interfaced with two FPGA of different families one is Virtex 6 and the other is Spartan 6 FPGA. The address register is used in the communication network to store data bit and to manipulate the stored bit [2]. In our design, we are scaling the voltage of address register from $0.75 \mathrm{~V}$ to $2.00 \mathrm{~V}$ for Virtex 6 FPGA and $0.75 \mathrm{~V}$ to $3.00 \mathrm{~V}$ for Spartan 6 FPGA for analysing the different on chips power which is fabricated on this device. The RTL schematic and technology schematic of the address register is shown in Fig. 1 and 2 respectively.

Revised Manuscript Received on July 18, 2019.

Bishwajeet Pandey, Center of Energy Excellence, Gyancity Research Lab, Motihari, Bihar, India(email: gyancity@gyancity.com)

Keshav Kumar, Chitkara University Institute of Engineering \& Technology, Chitkara University, Punjab, India(email: keshav.kumar@chitkara.edu.in)

Shabeer Ahmad, Gran Sasso Science Institute, L'Aquila, Italy (email: shabeer.ahmad@gssi.it)

Amit K Pandit, The Shri Mata Vaishno Devi University, Katra, Jammu and kashmir India(email: amit.pandit@smvdu.ac.in)

Deepa Singh, ABV-IIITM, Gwalior,Madhya Pradesh, India(email: deepa@iiitm.ac.in)

D M Akbar Hussain, Department of Energy Technology, Aalborg University, Esbjerg, Denmark(email: akh@et.aau.dk).

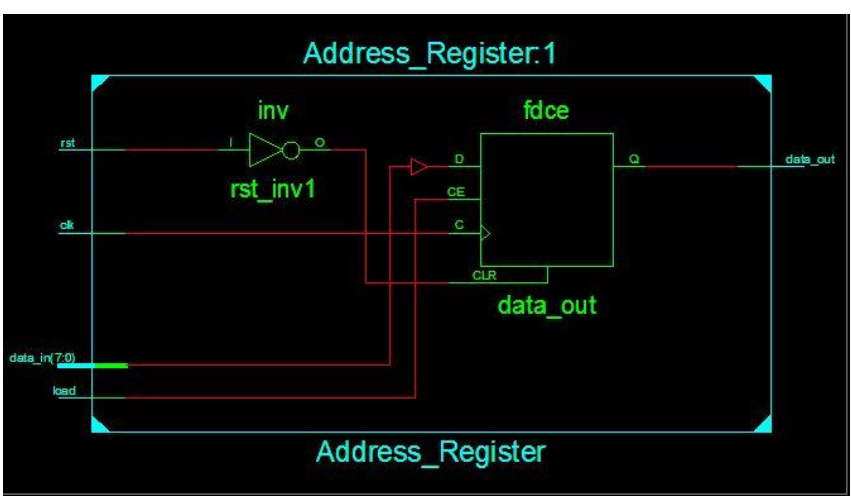

Figure 1. RTL schematic of the address register.

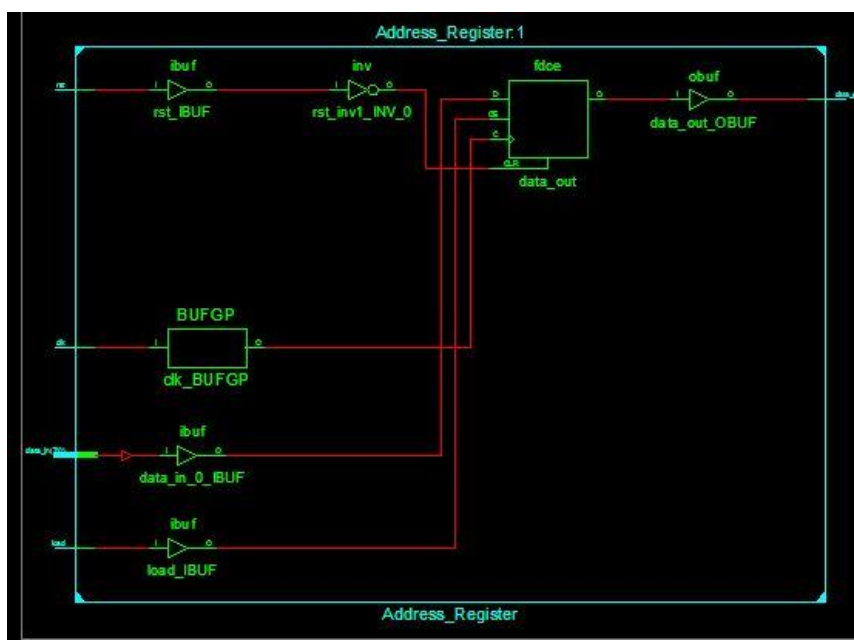

Figure 2. Technology schematic of the address register.

\section{EXPERIMENTAL SETUP}

We performed our experiment on Xilinx ISE Design Suite and the code of address register is written in Verilog Hardware Language. The power consumption of address register is analysed on X Power analyser tool. The ambient temperature of both the FPGA is $50 \mathrm{oC}$ and has an airflow of 250 LFM [Linear Feet Meter].

\section{RELATED WORK}

I.Kabin et.al [3] designed FPGA implementation of ECC. In this work, authors discussed hardware implementation of 


\section{LEAKAGE POWER CONSUMPTION OF ADDRESS REGISTER INTERFACING WITH DIFFERENT FAMILIES OF FPGA}

address bits on FPGA. W.Tang et.al [4] designed hardware design of genetic algorithm on FPGA. In this work author designed parallel configuration with PCI board. M.Chmiel

[5] implemented Central Processing Unit with Virtex 4 FPGA. This work represents a specific timer and control hardware solution. B. Pandey et.al [6] worked on designing a low voltage and low power VLSI circuit on FPGA. They used Virtex 6 FPGA and RAM-UART to analyse power reduction. B. Pandey et.al [7] implemented energy efficient ALU [Arthemetic Logic Unit] on 40nm FPGA. Spartan 3 and Virtex 6 FPGA are used to analyse dynamic power reduction with LVCMOS12. E.B.Kavun et.al [8] designed FPGA implementation of lightweight cypher PRESENT on Xilinx Spartan XC3S50 device. But in our work we have interfaced address register with two FPGA, Virtex 6 and Spartan 6 to analyse the leakage power consumption.

\section{POWER ANALYSIS \& RESULTS}

A. Power analysis of Address Register interfaced with Virtex 6 FPGA.

When the voltage of Virtex 6 FPGA scaled from $0.75 \mathrm{~V}$ to $2 \mathrm{~V}$, it is observed that there is only consumption of leakage power in the address register. All the other on chips power e.g. clocks, logic, signal, I/O are negligible. As voltage increases, there is an increment in leakage power. Thus we can state that lower the voltage lesser will be the consumption of leakage power. The power chart of Virtex 6 FPGA is shown in Table 1 . The magnitude of $1.75 \mathrm{~V}$ is the last operating voltage of Virtex 6 FPGA, beyond this voltage i.e. at $2 \mathrm{~V}$ the interfacing of address register with Virtex 6 FPGA burns out. The power v/s voltage figure of Virtex 6 FPGA is shown in Fig. 3.

Table 1. Leakage power with Virtex 6 FPGA.

\begin{tabular}{|c|c|}
\hline Voltage Value (V) & Leakage Power (W) \\
\hline 0.75 & 0.887 \\
\hline 1.00 & 1.293 \\
\hline 1.25 & 2.396 \\
\hline 1.50 & 5.625 \\
\hline 1.75 & 16.596 \\
\hline 2.00 & 39.641 \\
\hline
\end{tabular}

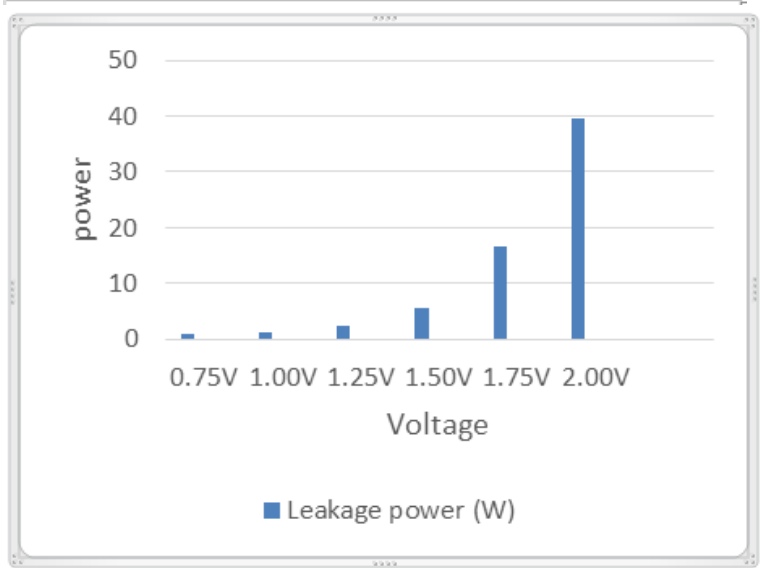

Figure 3. Power V/S Voltage with Virtex 6 FPGA.
B. Power analysis of Address Register interfaced with Spartan 6 FPGA.

Interfacing of Virtex 6 with address register burns at $2 \mathrm{~V}$, but in case of Spartan 6 FPGA the interfacing burns at $3 \mathrm{~V}$. In the case of Spartan 6 FPGA also as the value of voltage increases, there is an increment in leakage power. The power chart is shown in Table 2 and power v/s voltage figure is shown in Fig. 4.

Table 2. Leakage power with Spartan 6 FPGA.

\begin{tabular}{|c|c|}
\hline Voltage Value (V) & Leakage Power (W) \\
\hline 0.75 & 0.010 \\
\hline 1.00 & 0.011 \\
\hline 1.25 & 0.015 \\
\hline 1.50 & 0.024 \\
\hline 1.75 & 0.049 \\
\hline 2.00 & 0.115 \\
\hline 3.00 & 20.125 \\
\hline
\end{tabular}

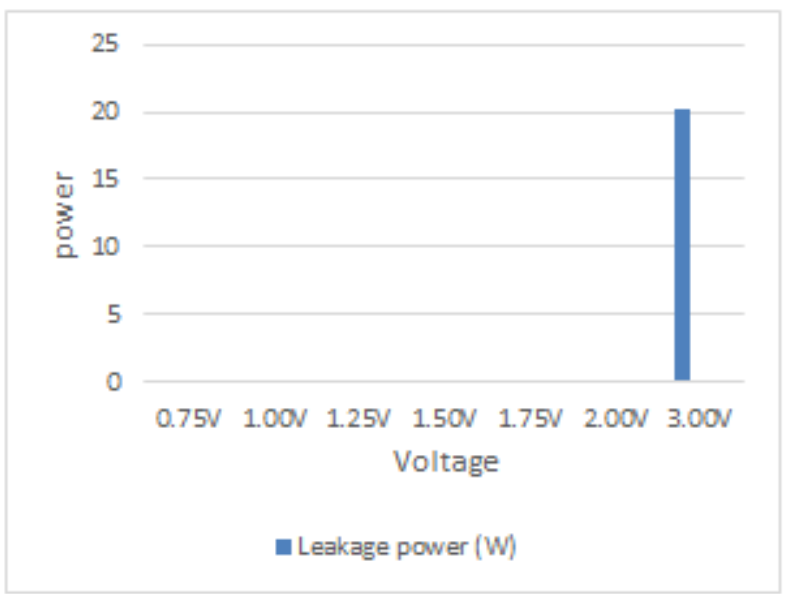

Figure 4. Power V/S Voltage with Spartan 6 FPGA.

C. Power Analysis of Virtex 6 V/S Spartan 6 FPGA.

The leakage power consumption of Spartan 6 FPGA is lower than Virtex 6 FPGA at every value of voltage. Also, Spartan 6 FPGA gives more voltage range of operating address register since it burns at $3 \mathrm{~V}$ were as Virtex 6 at $2 \mathrm{~V}$. The power comparison table of both FPGA is shown in Table 3. 
Table 3. Leakage power of Virtex 6 V/S Spartan 6.

\begin{tabular}{|c|c|c|}
\hline Voltage (V) & $\begin{array}{c}\text { Leakage power } \\
\text { (Virtex 6) }\end{array}$ & $\begin{array}{c}\text { Leakage power } \\
\text { (Spartan 6) }\end{array}$ \\
\hline 0.75 & 0.887 & 0.010 \\
\hline 1.00 & 1.293 & 0.011 \\
\hline 1.25 & 2.396 & 0.015 \\
\hline 1.50 & 5.625 & 0.024 \\
\hline 1.75 & 16.596 & 0.049 \\
\hline 2.00 & 39.641 & 0.115 \\
\hline 3.00 & 0.000 & 20.125 \\
\hline
\end{tabular}

\section{CONCLUSION}

From the power analysis of both the FPGA, it is observed that Spartan 6 FPGA consumes the least amount of power. Spartan 6 takes $98.87 \%, 99.14 \%, 99.37 \%, 92.90 \%, 99.70 \%$, and $99.70 \%$ less leakage power than Virtex 6 FPGA at $0.75 \mathrm{~V}, 1.00 \mathrm{~V}, 1.25 \mathrm{~V}, 1,50 \mathrm{~V}, 1.75 \mathrm{~V}$, and $2.00 \mathrm{~V}$ respectively. On interfacing address register with Virtex 6 and Spartan 6 FPGA on Xilinx ISE Design Suite, we found that Spartan 6 is a most power-efficient device at all values of voltage and also gives a wide range of voltage value for the operation.

\section{FUTURE SCOPE}

This work describes the interfacing of address register with Virtex 6 and Spartan 6 FPGA. But we can also interface address register with other FPGAs like Cyclone FPGA, Artix-7 FPGA, and Kintex-7 FPGA etc. for analyzing the power. We can also scale the current value, frequency value and change $\mathrm{I} / \mathrm{O}$ standards to observe the variation in the power consumption that will be helpful in green communication.

\section{REFERENCES}

1. Cause and Soultion of Global Energy Crisis, www.conserve-energy-future.com/causes-and-solutionto-the-global-energy-crisis.php, Last Accessed on 5th May 2019

2. Definition of Address Register, www.definitions.net/definition/memory+address+register , Last Accessed on 5th May 2019

I. Kabin, D. Kreiser, Z. Dyka, and P. Langendoerfer. "FPGA Implementation of ECC: Low-Cost Countermeasure against Horizontal Bus and Address-Bit SCA." In 2018 International Conference on ReConFigurable Computing and FPGAs (ReConFig), pp. 1-7. IEEE, 2018.

3. W. Tang, and L.Yip. "Hardware implementation of genetic algorithms using FPGA." In The 2004 47th Midwest Symposium on Circuits and Systems, 2004. MWSCAS'04., vol. 1, pp. I-549. IEEE, 2004.

4. M. Chmiel, J. Mocha, E. Hrynkiewicz, and A. Milik. "Central processing units for PLC implementation in Virtex-4 FPGA." IFAC Proceedings Volumes 44, no. 1 (2011): 7860-7865.

5. B. Pandey, and R. Kumar. "Low voltage DCI based low power VLSI circuit implementation on FPGA." In 2013
IEEE Conference on Information \& Communication Technologies, pp. 128-131. IEEE, 2013..

6. B. Pandey, J. Yadav, Y. K. Singh, R. Kumar, and S Patel. "Energy efficient design and implementation of ALU on 40nm FPGA." In 2013 International Conference on Energy Efficient Technologies for Sustainability, pp. 45-50. IEEE, 2013.

7. E.B. Kavun, and T. Yalcin. "RAM-based ultralightweight FPGA implementation of PRESENT." In 2011 International Conference on Reconfigurable Computing and FPGAs, pp. 280-285. IEEE, 2011. 